\title{
Long-term consequences of mild traumatic brain injury ${ }^{\dagger}$
}

Roberto J. Rona

\begin{abstract}
Summary
A debate has ensued about the long-term consequences of mild traumatic brain injury, the 'signature injury' of the Iraq and Afghanistan Wars. Most epidemiological studies have found that mild traumatic brain injury is unrelated to unspecific post-concussion symptoms based on self-reported symptoms. A longitudinal study, in this issue of the
\end{abstract}

Journal, using objective tests has demonstrated that mild traumatic brain injury has limited lasting neuropsychological consequences

\section{Declaration of interest}

None.
Roberto J. Rona is Professor of Public Health. He is involved in studies to assess the prevalence and aetiology of mental disorders, and the effectiveness of screening for mental illnesses in the UK military.

Military service is inherently a dangerous occupation. Peace enforcement and war missions increase the chance of traumatic experiences that may lead to psychological problems. Blasts, shrapnel, falls and road accidents could cause mild traumatic brain injury, i.e. concussion. Mild traumatic brain injury has been labelled the 'signature injury' of the Iraq and Afghanistan Wars and, indeed, by 2008 about 320000 personnel may have experienced traumatic brain injury; the great majority, mild traumatic brain injury. ${ }^{1}$ The phrase signature injury reflects the particular hazards in the Iraq and Afghanistan Wars from improvised explosive devices, but it is as well to remember that explosive devices were also responsible for the signature injury of the First World War, namely shellshock, characterised by non-specific symptoms attributed to the concussive or toxic effects of projectile shells. ${ }^{2}$

Mild traumatic brain injury is recognised in military personnel who have suffered head injuries followed by a brief loss of consciousness or symptoms of altered mental status. Mild traumatic brain injury and long-term post-concussion symptoms such as headaches, dizziness, memory problems, balance problems, lack of concentration, ringing in the ears and irritability are possible consequences of exposure to blast. However, another possible consequence is post-traumatic stress disorder (PTSD). The same event can give rise to mild traumatic brain injury and subsequently, in some, to post-concussion symptoms, presumed to be the consequence of organic injury to the brain, and PTSD the consequence of psychological injury to the mind.

That logic assumes that the same aetiological factor could have been responsible for PTSD and mild traumatic brain injury, but in practice things are not so simple. The three largest US military studies found that between 33 and 39\% of those who reported probable mild traumatic brain injury also have probable PTSD. ${ }^{3}$ This figure rises to $44 \%$ in individuals reporting loss of consciousness and decreases to $27 \%$ in those with altered mental status but no loss of consciousness. ${ }^{4}$ Post-traumatic stress disorder in those with self-reported mild traumatic brain injury in the UK military is $15 \%$ in a sample in which the great majority with mild traumatic brain injury had altered mental status but not loss of

'See pp. 186-192, this issue. consciousness. ${ }^{5}$ This prevalence of probable PTSD among personnel with mild traumatic brain injury in the UK forces is in line with the difference in the prevalence of PTSD in deployed US and UK personnel, which is about $15 \%$ and $4 \%$ respectively. ${ }^{4,6}$ Depression is also more common in those who have reported mild traumatic brain injury. ${ }^{4,7}$ Mild traumatic brain injury, PTSD and depression tend to cluster together in deployed personnel. Alcohol misuse is also more frequent in those who reported mild traumatic brain injury. ${ }^{5}$

\section{Mild traumatic brain injury and its long-term consequences}

It is currently being debated as to whether mild traumatic brain injury has enduring consequences in terms of post-concussion symptoms in the military. ${ }^{8}$ The case that mild traumatic brain injury may lead to long-term disability was recently made by a study that used diffusion tensor imaging and found an association between blast-related mild traumatic brain injury and imaging abnormalities which persist in some individuals. ${ }^{9}$ However, these researchers did not relate their finding to post-concussion symptoms and the studied group represented a highly selected sample of personnel with mild traumatic brain injury who were evacuated to Germany, while the great majority was not. ${ }^{10}$ Structural and functional studies have not clearly established causality with persistent post-concussion symptoms. ${ }^{7}$ The consequences of mild traumatic brain injury during deployment have been particularly difficult to study so far because the symptoms associated with mild traumatic brain injury as a result of a blast are usually reported many months after the event, so are subject to reporting bias. In addition, post-concussion symptoms are non-specific, ${ }^{4,5}$ many of the symptoms are common to depression, PTSD and other conditions, and people who report non-specific post-concussion symptoms also report an excess of other physical symptoms. ${ }^{4,5}$ In contrast to studies in athletes in which the presence of trained healthcare staff during sports events can ensure reliable information on mild traumatic brain injury, ${ }^{11}$ epidemiological studies of deployed personnel rely on self-report of injury events. The level of reliability of these reports is uncertain, as shown in a longitudinal study in which mild traumatic brain injury had a prevalence of $9 \%$ when assessed a month before the end of deployment, but $22 \%$ a year after returning from deployment in the same personnel. ${ }^{12}$ The great majority of self-reported cases of mild traumatic brain injury do not access medical services soon after the event, which further increases the level of uncertainty. 


\section{Analytical approaches to the debate so far}

The statistical strategy to assess post-concussion symptoms following mild traumatic brain injury in military studies has been to compare each non-specific concussion symptom in mild traumatic brain injury cases and in controls (who had injuries but not mild traumatic brain injury) and adjust for concomitant PTSD, other physical symptoms and depression. ${ }^{4,5}$ Some studies have used, as controls, individuals without mild traumatic brain injury but not restricted to those with other injuries. ${ }^{12}$ Other studies have not adjusted for PTSD, but considered that a greater joint effect of PTSD and mild traumatic brain injury on postconcussion symptoms was sufficient to demonstrate the long-term effect of mild traumatic brain injury. ${ }^{13}$ Most studies, including a longitudinal study, have shown that for most symptoms there is no association between mild traumatic brain injury and postconcussion symptoms after adjustments for $\mathrm{PTSD}^{4,12}$ or adjustment for multiple physical symptoms and PTSD. ${ }^{5}$ However, headaches and dizziness remained associated with mild traumatic brain injury. ${ }^{4,5}$ Brenner and colleagues reported that mild traumatic brain injury and PTSD together are more strongly associated with post-concussion symptoms than either condition separately. ${ }^{13}$ Such results would be expected in a group of individuals with both mild traumatic brain injury and PTSD. Their view would have been more appealing had they shown an interaction between PTSD and mild traumatic brain injury on post-concussion symptoms. On the other hand, one should be aware of the dangers of adjusting for PTSD, as some postconcussion symptoms such as sleeping difficulties, fatigue and lack of concentration are also symptoms of PTSD. This leaves researchers in a dilemma as to whether to disregard these symptoms when assigning individuals to PTSD in the analysis.

\section{New contribution to the debate}

Vasterling and colleagues' article in the current issue of the Journal is to be welcomed because they explore the possible neuropsychological consequences of mild traumatic brain injury rather than a list of non-specific possible symptoms of concussion. ${ }^{14}$ They assessed the consequences of mild traumatic brain injury and its interrelation with PTSD and depression using an objective battery of neuropsychological tests in 760 US personnel deployed to Iraq. The battery of measurements included several aspects of memory, sustained attention, aspects of learning and cognitive inhibition, and reaction time. They included measures to assess processing speed, which has been previously found to be diminished in those who had sustained mild traumatic brain injury with or without PTSD. ${ }^{14}$ Neuropsychological tests were administered before and after returning from deployment. Thus, the researchers were able to account in the analysis for pre- deployment neuropsychological status and therefore account for the pre-deployment estimate of the neuropsychological outcomes.

The results were that out of the 13 neuropsychological outcomes in the follow-up assessment, mild traumatic brain injury was associated only with visual reproductions (immediate recall), even before adjusting for PTSD or depression, whereas PTSD and depression were associated with several neuropsychological outcomes, including processing speed, even after adjusting for mild traumatic brain injury.

There are some limitations to Vasterling et al's study. ${ }^{14}$ The study included as cases of mild traumatic brain injury only individuals with loss of consciousness following blast-related injury. Cases of mild traumatic brain injury with altered mental state but no loss of consciousness may have been included as part of the control group in the analysis. This might reduce the contrast between mild traumatic brain injury and controls. This is important because those who sustained mild traumatic brain injury with altered mental status only are not proper controls and are potentially liable to represent a group with neuropsychological symptoms. The control group comprised anyone without mild traumatic brain injury (loss of consciousness), whereas other studies include in the control group only individuals who suffered injury but not mild traumatic brain injury. This has been considered necessary in studies of mild traumatic brain injury because some of the post-concussion symptoms could be related to injury in the absence of mild traumatic brain injury and would be more appropriate to discount 'background noise'. Vasterling et al's approach is understandable because it increased the statistical power and had they restricted the analysis to those who reported injury, the study would have been underpowered. However, such a limitation would have increased the chance of finding differences between the two groups and reduced the chance of false negative findings.

The remaining problem in studies of mild traumatic brain injury in deployed populations is that they rely on individuals' self-reports using a poorly validated tool. Further progress could be achieved if these reports were accredited by trained healthcare staff near to the time of the event. However, this is the counsel of perfection in an operational environment.

Overall, Vasterling and colleagues add to the evidence that mild traumatic brain injury, as reported by military personnel following deployment, has minimal lasting consequences for the majority, even if it can still not exclude such an effect for a small number, admittedly rather fewer than claimed elsewhere. The advice of Vasterling et al is appropriate - healthcare providers should pay attention to symptoms of depression and PTSD in any veterans reporting mild traumatic brain injury. Healthcare staff can also reassure those who report having sustained mild traumatic brain injury in a blast-related event of a good long-term prognosis.

Roberto J. Rona, PhD, FFPH, King's Centre for Military Health Research, Weston Education Centre, King's College London, Cutcombe Road, London SE5 9RJ, UK. Email: roberto.rona@kcl.ac.uk

First received 5 Mar 2012, final revision 25 May 2012, accepted 23 Jul 2012

\section{References}

1 Tanielian T, Jaycox LH (eds). Invisible Wounds of War: Psychological and cognitive Injuries, their Consequences, and Services to Assist Recovery. RAND Corporation, 2008.

2 Jones E, Fear NT, Wessely S. Shell shock and mild traumatic brain injury: a historical review. Am J Psychiatry 2007; 164: 1641-5.

3 Carlson KF, Kehle SM, Meis LA, Greer N, MacDonald R, Rutks I, et al. Prevalence, assessment, and treatment of mild traumatic brain injury and posttraumatic stress disorder: a systematic review of the evidence. J Head Trauma Rehabil 2011; 26: 106-15.

4 Hoge CW, McGurk D, Thomas JL, Cox AL, Engel CC, Castro CA. Mild traumatic brain injury in U.S. Soldiers returning from Iraq. N Engl J Med 2008; 358 : 453-63.

5 Rona RJ, Jones M, Fear NT, Hull L, Murphy D, Machell L, et al. Mild traumatic brain injury in UK military personnel returning from Afghanistan and Iraq: cohort and cross-sectional analyses J Head Trauma Rehabil 2012; 27: 33-44.

6 Fear NT, Jones M, Murphy D, Hull L, Iversen A, Coker B, et al. What are the consequences of deployment to Iraq and Afghanistan on the mental health of the UK Armed Forces? A cohort study. Lancet 2010; 375: 1783-97.

7 Lange RT, Iverson GL, Rose A. Depression strongly influences postconcussion symptom reporting following mild traumatic brain injury. J Head Trauma Rehabil 2011; 26: 127-37. 
8 Hoge CW, Goldberg HM, Castro CA. Care of war veterans with mild traumatic brain injury - flawed perspectives. N Engl J Med 2009; 360: 1588-91.

9 MacDonald $C L$, Johnson AM, Cooper D, Nelson EC, Werner NJ, Shimony JS, et al. Detection of blast-related traumatic brain injury in US military personnel. New Engl J Med 2011; 364: 2091-100.

10 Hoge CW, Castro CA. Blast related Traumatic Brain Injury in US military personnel (letter). New Engl J Med 2011; 365: 860.

11 McCrea MA. Mild Traumatic Brain Injury and Postconcussion Syndrome. The New Evidence Base for Diagnosis and Treatment (Oxford Workshop Series: American Academy of Clinical Neuropsychology). Oxford University Press, 2008
12 Polusny MA, Kehle SM, Nelson NW, Erbes CR, Arbisi PA, Thuras P. Longitudinal effects of mild traumatic brain injury and posttraumatic stress disorder comorbidity on postdeployment outcomes in National Guard soldiers deployed to Iraq. Arch Gen Psychiatry 2011; 68: 79-89.

13 Brenner LA, Ivins BI, Schwab K, Warden D, Nelson LA, Jaffee M, et al. Traumatic brain injury, posttraumatic stress disorder, and postconcussive symptom reporting among troops returning from Iraq. J Head Trauma Rehabil 2010; 25: 307-12.

14 Vasterling JJ, Brailey K, Proctor SP, Kane R, Heeren T, Franz M. Neuropsychological outcomes of mild traumatic brain injury, post-traumatic stress disorder and depression in Iraq-deployed US Army soldiers. Br J Psychiatry 2012; 201: 186-92.

\title{
The 'autoimmune' mind
}

\author{
Shabbir Amanullah
}

From a therapeutic point of view, psychiatry as a specialty is in an unenviable position. If, despite extensive interventions, a patient takes his or her own life, the physician is often perceived as having failed that person. Rarely is a consultant oncologist challenged about a non-responsive patient: 'surely, there must be something you could have done differently?' Despite rapid advances across medicine, psychiatrists frequently wonder why placebos work 'just as well' in some disorders.

Rethinking how the mind, in all its complexity and might, is deeply fallible may help us. In depression, negative automatic thoughts prevent people from functioning normally; in post-traumatic stress disorder, a distressing memory can almost take on a 'life of its own'. In obsessive-compulsive disorder, one is confronted with a persistence of such thoughts despite insight that they are 'silly'. As phenomena, the thoughts appear to be 'against the mind'

That the mind chooses to attack itself is in itself analogous to the body's autoimmune reaction. We assume that childhood events shape our subsequent reactions to events. Why then do apparently well-adapted individuals struggle or indeed develop depression in later life? From a non-biological perspective, how do some of the most accomplished people have low self-esteem? Evidence and clinical experience support a combination of different interventions for psychiatric disorders. One target is the immediate 'inflammatory' response, and perhaps psychoactive medications for agitation/anxiety do that, but parallel to this, antidepressants and antipsychotics may act like immune suppressants - as thought suppressants. We know that psychotherapeutic approaches help, but we may need to add meditation to our interventions in an effort to suppress extraneous unwanted thoughts, as alternatives to the conventional combination of psychotherapy and psychopharmacology. 